\title{
Ecotoxicity and Preliminary Risk Assessment of Nonivamide as a Promising Marine Antifoulant
}

\author{
Sujing Liu, ${ }^{1,2}$ Jun Zhou, ${ }^{1}$ Xuanxuan Ma, ${ }^{3}$ Ying Liu, ${ }^{3}$ Xing Ma, ${ }^{1,2}$ and Chuanhai Xia ${ }^{1,3}$ \\ ${ }^{1}$ Yantai Institute of Coastal Zone Research, Chinese Academy of Sciences, Yantai 264003, China \\ ${ }^{2}$ University of Chinese Academy of Sciences, Beijing 100049, China \\ ${ }^{3}$ School of Resources and Environmental Engineering, Ludong University, Yantai 264025, China
}

Correspondence should be addressed to Ying Liu; liuyingldu@hotmail.com and Chuanhai Xia; chxia_ldu@hotmail.com

Received 28 February 2016; Accepted 5 April 2016

Academic Editor: Jun Wu

Copyright (C) 2016 Sujing Liu et al. This is an open access article distributed under the Creative Commons Attribution License, which permits unrestricted use, distribution, and reproduction in any medium, provided the original work is properly cited.

\begin{abstract}
The unclear environmental performance of nonivamide limits its application as a marine antifoulant. In this study, the natural degradation of nonivamide was studied in seawater and tap water. The half-life was $5.8 \mathrm{~d}, 8.8 \mathrm{~d}, 12.2 \mathrm{~d}$, and $14.7 \mathrm{~d}$ in seawater and tap water in photolysis and biolysis, respectively. Furthermore, the ecotoxicity of nonivamide was assessed using marine microalgae, Chlorella vulgaris and Platymonas sp.; $\mathrm{EC}_{50,6 \mathrm{~d}}$ values on the growth of Chlorella vulgaris and Platymonas sp. were $16.9 \mathrm{mg} \mathrm{L}^{-1}$ and $19.21 \mathrm{mg} \mathrm{L}^{-1}$, respectively. The toxicity and environmental risk of nonivamide on microalgae were significantly decreased due to the natural degradation in seawater.
\end{abstract}

\section{Introduction}

Marine biofouling is caused by the adhesion of barnacles, macroalgae, and microbial slimes, which is a worldwide problem in marine systems $[1,2]$. When fouling organisms attach to a ship's hull, the increased hydrodynamic drag results in decreased speed, higher fuel consumption, and more frequent removal from service for hull cleaning [3-6]. Marine paints containing tributyltins (TBT) have played a major role in improving the shipping industry's economics. However, the persistence of TBT in the environment, combined with its toxicity towards certain marine and freshwater organisms, has led many governments to impose restrictions on its use $[7,8]$. The International Maritime Organization (IMO) has prohibited the application of organotin compounds which act as antifoulants in antifouling systems on ships since January 2003. A proposal has highlighted the need for safer alternatives of organotin compounds (MEPC 42/22, 1998). The ideal replacement will have a broad spectrum of activity against a diverse population of fouling organisms and provide up to five years of antifouling performance without impacting target organisms. Since risk is directly related to environmental concentration, the antifoulant should also rapidly degrade to nontoxic compounds when released into the aquatic environment.

Now, the general strategy to find safer alternatives was to identify natural products with good antifouling activity and explore their lethality, as well as the activity and lethality of structurally related and commercially available chemicals. The literature has revealed that capsaicin (CAS: 404-864 ), the active component of hot chili peppers (Capsicum), could effectively inhibit zebra mussel byssal attachment [9]. Additionally, the structure-activity relationships and mussel adhesion inhibitory activities of capsaicinoid members and their synthetic derivates have also been reported [10-12]. It was found that capsaicin and nonivamide (CAS: 2444-46-4, Scheme 1) showed the most effective antifouling activities.

Capsaicin, as a safer alternative of organotin compounds, has been used in ship antifouling paints in China according to the National Environmental Protection Standard of the People's Republic of China (HJ 2515-2012). Nonivamide, as a synthesized derivate of natural capsaicin, also has an effective antifouling activity [10-13]. Compared to capsaicin, it is more suitable for large-scale synthesis and industrial application owing to its lower price. However, to our knowledge, the ecotoxicity and environmental risk of nonivamide, as a synthetic 
<smiles>COc1cc(CNC(=O)CCCC/C=C/C(C)C)ccc1O</smiles>

Capsaicin<smiles>CCCCCCCCC(=O)NCc1ccc(O)c(OC)c1</smiles>

Nonivamide

Scheme 1: The structure of capsaicin and nonivamide.

chemical, are not clear, which will limit the application of nonivamide for antifouling paints.

For assessing the potential ecological risks of a biocide, algal toxicity assay is generally applied. The chief reason lies in the short life cycle, simple cultural requirements for most algae species, and their role as the primary producer in the nature [14]. However, because of the algal batch-type differences, algal toxicity assay is always lacking repetitiveness [15]. To overcome this defect, two different algal species, $C$. vulgaris and Platymonas sp., were together used in this study. C. vulgaris is a unicellular microalgae, while Platymonas sp. has complex flagella, a larger cell size, and multiple cellular contents, which have a longer reproductive cycle, lower growth rate, and stronger individual surviving ability [16]. The widely physiological differences of the two algae species lead to distinguished ecological performances, and from the perspective of ecology, they could be, respectively, considered as $r$-selected species and $K$-selected species [17]. $P$. tricornutum has previously been proved to be sensitive to nonivamide in our study $\left(\mathrm{EC}_{50,4 \mathrm{~d}}, 5.1 \mathrm{mg} \mathrm{L}^{-1}\right)$ [14] and was used to evaluate the toxicity nonivamide degradation products.

Regulatory agencies in the US, Europe, Australia, and other countries require applicants to perform various studies according to specific guidelines designed to determine environmental degradation rates under abiotic (hydrolysis and/or photolysis) and biotic (aerobic and/or anaerobic aquatic metabolism) conditions before a biocide is approved for use as an active ingredient. In this paper, the ecotoxicity and preliminary risk of nonivamide were studied and assessed in aquatic systems as a promising antifoulant.

\section{Experimental}

2.1. Biological Material and Culture Conditions. Platymonas sp., C. vulgaris, and P. tricornutum were provided by the Institute of Oceanology, Chinese Academy of Sciences. The algae were grown in axenic conditions, in $\mathrm{f} / 2$ medium based on autoclaved natural seawater at $20^{\circ} \mathrm{C}$ and light photon intensity of $48 \mu \mathrm{mol} \mathrm{m}{ }^{-2} \mathrm{~s}^{-1}$ with a $12: 12 \mathrm{~h}$ light : dark cycle. All cultures were shaken twice a day and cultured to the exponential phase before inoculation in the following experiment.

2.2. Algal Toxicity Assessment. The tested alga Platymonas sp. and C. vulgaris were chosen for algal toxicity assay. The growth inhibition effect of nonivamide on alga was assayed, and $\mathrm{EC}_{50}$ (the minimum effective inhibition concentration of nonivamide) was calculated by SigmaPlot 10.0 using logistic curve fitting based on equation. The alga density was counted with a haemocytometer. The initial alga density (IAD) used for algal toxicity assay was $1.0 \sim 2.0 \times 10^{5}$ cells $\mathrm{mL}^{-1}$ (for Platymonas sp.) and 1.0 2.0 $\times 10^{5}$ cells $\mathrm{mL}^{-1}$ (for C. vulgaris).

The toxicity of nonivamide with different exposure time was tested to reflect the algal toxicity of natural degradation products of nonivamide. P. tricornutum was chosen for toxicity evaluation for nonivamide degradation products. Five groups of $100 \mathrm{mg} \mathrm{L}^{-1}$ nonivamide were exposed to sunlight, and every week a group of samples were taken back and kept in dark at $4^{\circ} \mathrm{C}$ until the algal toxicity assay is applied. One group of nonivamide kept in dark was set as the control. For algal toxicity assay, the nonivamide solution $\left(100 \mathrm{mg} \mathrm{L}^{-1}\right)$ will be diluted 20 -fold with $\mathrm{f} / 2$ culture medium (IAD, 1.0 2.0 $\times 10^{5}$ cells $\left.\mathrm{mL}^{-1}\right)$. $\mathrm{OD}_{680}$ values of samples were measured to characterize the alga concentration. In order to easily compare, the growth rates of algae were shown in the form of specifical growth rates of the algae in the study, which was calculated by the following formula:

$$
\begin{aligned}
& \text { Specifical growth rate } \\
& \qquad=\frac{\mathrm{OD}_{680}(\text { Treated with nonivamide })}{\mathrm{OD}_{680}(\text { Control })} .
\end{aligned}
$$

All experiments were performed at least in triplicate.

2.3. The Evaluation of the Half-Life of Natural Degradation of Nonivamide. The natural degradation studies of nonivamide were conducted under both abiotic (hydrolysis and/or photolysis) and biotic conditions in natural water (seawater and tap water) including nonivamide (concentration of $50 \mathrm{mg} \mathrm{L}^{-1}$ ). In order to evaluate the abiotic degradation, biolysis effects were eliminated by adding sodium azide into the natural seawater and tap water. Samples degraded in the dark were set as the negative control. The solvent loss caused by evaporation in the experiment process was refilled back. The degradation half-life of nonivamide could be calculated according to the obtained calibration equations.

\section{Results and Discussion}

3.1. Toxicity of Nonivamide on Algal Growth. The specific growth rates of nonivamide-treated C. vulgaris and Platymonas sp. were shown in Figure 1. The growth of C. vulgaris 


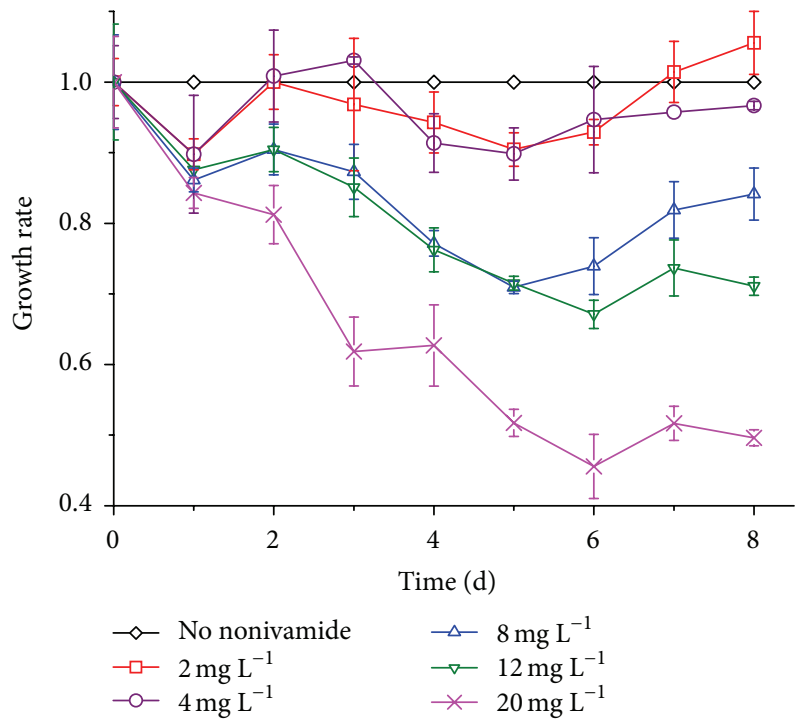

(a)

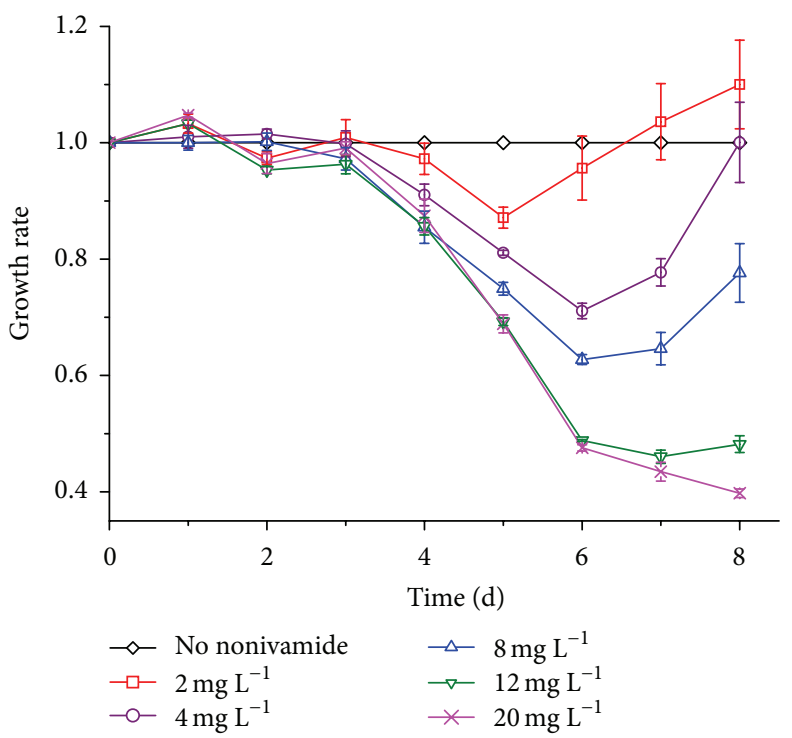

(b)

FIGURE 1: Growth inhibitory effects of nonivamide on (a) Chlorella vulgaris and (b) Platymonas sp. All error bars indicated SE of the three replicates.

would be inhibited if treated with nonivamide concentration higher than $4 \mathrm{mg} \mathrm{L}^{-1}$ (shown in Figure 1(a)). Based on the growth rates during the assay, $\mathrm{EC}_{50}$ values were calculated as follows: $18.3 \mathrm{mg} \mathrm{L}^{-1}$ at $6 \mathrm{~d}$ and $17.1 \mathrm{mg} \mathrm{L}^{-1}$ at $8 \mathrm{~d}$. Based on Figure 1(b), $\mathrm{EC}_{50}$ values were calculated as follows: $11.0 \mathrm{mg} \mathrm{L}^{-1}$ at $6 \mathrm{~d}$ and $10.7 \mathrm{mg} \mathrm{L}^{-1}$ at $8 \mathrm{~d}$. The results indicated that nonivamide could significantly inhibit the growth of the algae, and the inhibition effect would be more significant with the extension of exposure time. It was suggested that nonivamide might be a promising antifouling agent to marine microalgae. Additionally, when Platymonas sp. and C. vulgaris were treated with nonivamide at lower concentrations (2-8 $\left.\mathrm{mg} \mathrm{L}^{-1}\right)$, it was clearly seen that the growth of the tested algae would be restored after 6 days later. It means that the tested algae could adapt to toxicant stress after an adjustment period.

3.2. The Half-Life of Natural Degradation of Nonivamide. The photolysis and/or biolysis characteristic of nonivamide in tap water and seawater was shown in Figure 2, and sodium azide was added to eliminate biolysis effects in some samples. Nonivamide exhibited the fastest degradation and a halflife of $5.8 \mathrm{~d}$ in seawater without sodium azide. The half-life in other groups was $8.8 \mathrm{~d}$ in seawater with sodium azide, $12.2 \mathrm{~d}$ in tap water without sodium azide, and $14.7 \mathrm{~d}$ in tap water with sodium azide, respectively. The results showed that nonivamide in seawater degraded much faster than in tap water, and biolysis also should play an important role in degradation process. Due to the rapid degradation of nonivamide in sunlight exposure in sea water, it suggested that nonivamide would be suitable for use in marine environment. of Nonivamide. In order to evaluate the ecotoxicity and

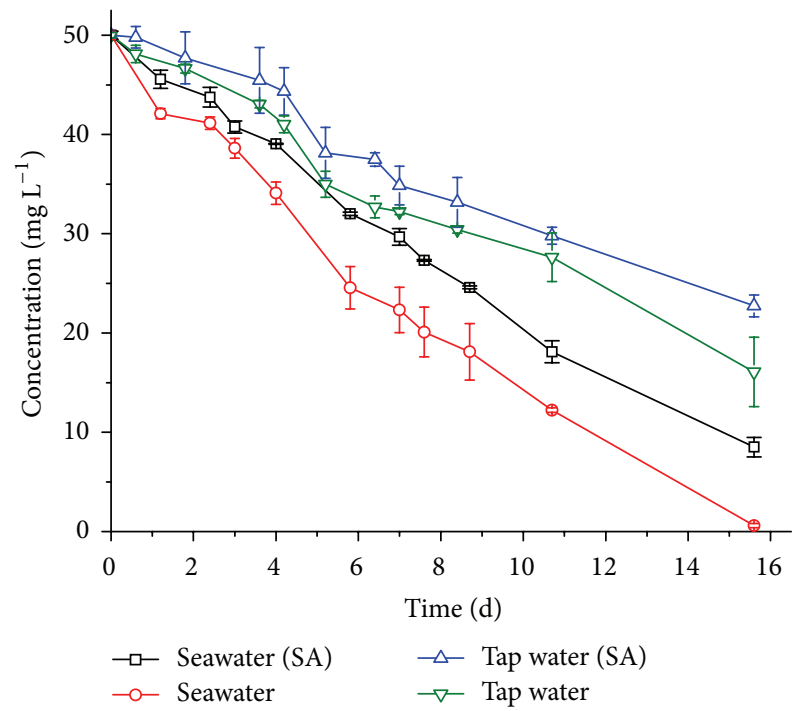

FIgURE 2: Degradation rates of nonivamide in seawater and tap waters. "SA" indicated that biolysis factor was eliminated by adding sodium azide. Error bars indicated SE of the three replicates.

environmental risk of natural degradation products of nonivamide, different sample groups of nonivamide exposed in sunlight from 1 week to 4 weeks were evaluated with P. tricornutum, which has been proved to be sensitive to nonivamide in our study $\left(\mathrm{EC}_{50,4 \mathrm{~d}}, 5.1 \mathrm{mg} \mathrm{L}^{-1}\right)$ [13]. As shown in Figure 3, the algal growth rates exposed in sunlight were similar to that of no nonivamide sample groups but were much higher than the growth rate exposed in dark. It suggested that the toxicity of nonivamide would be largely decreased after sunlight exposure. The results indicated that the ecotoxicity of nonivamide should be decreased possibly due to the natural degradation of nonivamide in field experiment, in which 


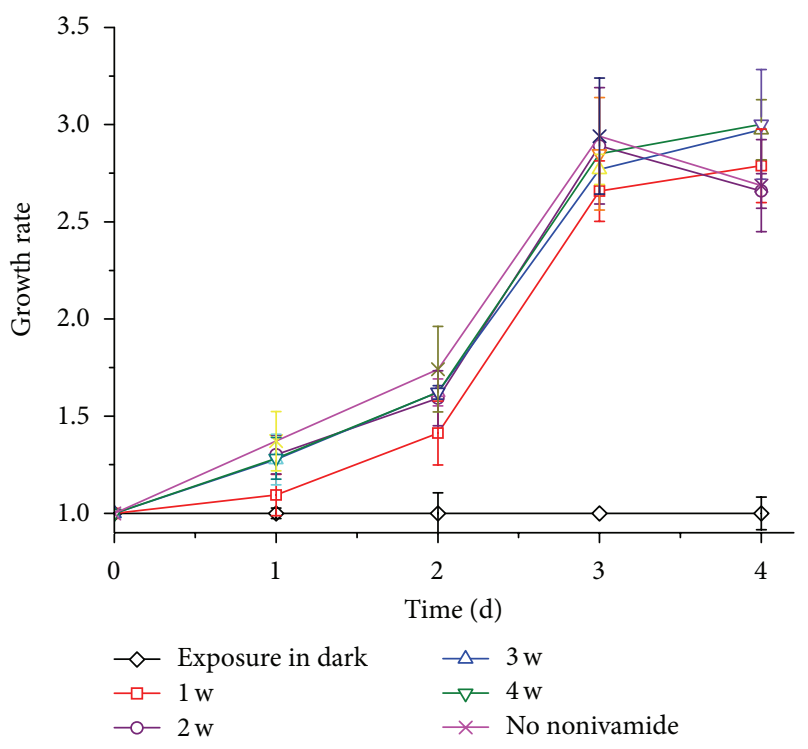

FIGURE 3: Growth rates of $P$. tricornutum treated with nonivamide with sunlight exposure (1,2,3, and 4 weeks). All error bars indicated $\mathrm{SE}$ of the three replicates.

some lowly toxic or nontoxic products would be generated in marine environment. It means that environmental risk of nonivamide was low as a marine antifoulant, and the natural degradation products of nonivamide hardly produced environmental risk in marine environment.

\section{Conclusions}

The natural degradation of nonivamide showed the rapid degradation rate in photolysis and/or biolysis, which indicated that nonivamide was easy to naturally degraded in marine environment. The ecotoxicity of nonivamide on marine microalgae would be significantly decreased due to natural degradation, which means that nonivamide and its degradation products should have little toxicity and low risk to microalgae in marine environment.

\section{Competing Interests}

The authors declare that there is no conflict of interests regarding the publication of this paper.

\section{Acknowledgments}

The present study was financially supported by the Project from Yantai Science and Technology Bureau (2014ZH084), the Cultivation Plan of Superior Discipline Talent Teams of Universities in Shandong Province, "the Coastal Resources and Environment Team for Blue-Yellow Area," and the National Natural Science Foundation of China (21377162).

\section{References}

[1] R. L. Townsin, “The ship hull fouling penalty," Biofouling, vol. 19, no. 2, pp. 9-15, 2003.
[2] I. Fitridge, T. Dempster, J. Guenther, and R. de-Nys, “The impact and control of biofouling in marine aquaculture: a review," Biofouling, vol. 28, no. 7, pp. 649-669, 2012.

[3] M. P. Schultz, J. A. Bendick, E. R. Holm, and W. M. Hertel, "Economic impact of biofouling on a naval surface ship," Biofouling, vol. 27, no. 1, pp. 87-98, 2011.

[4] M. P. Schultz, "Effects of coating roughness and biofouling on ship resistance and powering," Biofouling, vol. 23, no. 5, pp. 331341, 2007.

[5] M. P. Schultz, J. M. Walker, C. N. Steppe, and K. A. Flack, "Impact of diatomaceous biofilms on the frictional drag of fouling-release coatings," Biofouling, vol. 31, no. 9-10, pp. 759773, 2015.

[6] J. A. Finlay, M. P. Schultz, G. Cone, M. E. Callow, and J. A. Callow, "A novel biofilm channel for evaluating the adhesion of diatoms to non-biocidal coatings," Biofouling, vol. 29, no. 4, pp. 401-411, 2013.

[7] B. Antizar-Ladislao, "Environmental levels, toxicity and human exposure to tributyltin (TBT)-contaminated marine environment. A review," Environment International, vol. 34, no. 2, pp. 292-308, 2008.

[8] S. Mohr, R. Berghahn, W. Mailahn, R. Schmiediche, M. Feibicke, and R. Schmidt, "Toxic and accumulative potential of the antifouling biocide and TBT successor Irgarol on freshwater macrophytes: a pond mesocosm study," Environmental Science and Technology, vol. 43, no. 17, pp. 6838-6843, 2009.

[9] W. G. Cope, M. R. Bartsch, and L. L. Marking, "Efficacy of candidate chemicals for preventing attachment of zebra mussels (Dreissena polymorpha)," Environmental Toxicology and Chemistry, vol. 16, no. 9, pp. 1930-1934, 1997.

[10] H. Shi and L. M. Wang, "The preliminary studies on nontoxic capsaicin AF paint," Journal of Dalin Fisheries University, no. 4, pp. 322-325, 2005.

[11] Q. W. Xu, C. A. Barrios, T. Cutright, and B.-M. Z. Newby, "Evaluation of toxicity of capsaicin and zosteric acid and their potential application as antifoulants," Environmental Toxicology, vol. 20, no. 5, pp. 467-474, 2005.

[12] M.-B. Angarano, R. F. Mcmahon, D. L. Hawkins, and J. A. Schetz, "Exploration of structure-antifouling relationships of capsaicin-like compounds that inhibit zebra mussel (Dreissena polymorpha) macrofouling," Biofouling, vol. 23, no. 5, pp. 295305, 2007.

[13] J. Zhou, C. Yang, J. Wang et al., “Toxic effects of environmentfriendly antifoulant nonivamide on Phaeodactylum tricornutum," Environmental Toxicology and Chemistry, vol. 32, no. 4, pp. 802-809, 2013.

[14] A. D. P. Worgan, J. C. Dearden, R. Edwards, T. I. Netzeva, and M. T. D. Cronin, "Evaluation of a novel short-term algal toxicity assay by the development of QSARs and inter-species relationships for narcotic chemicals," QSAR \& Combinatorial Science, vol. 22, no. 2, pp. 204-209, 2003.

[15] J.-H. Lin, W.-C. Kao, K.-P. Tsai, and C.-Y. Chen, "A novel algal toxicity testing technique for assessing the toxicity of both metallic and organic toxicants," Water Research, vol. 39, no. 9, pp. 1869-1877, 2005.

[16] R. H. Peters, The Ecological Implications of Body Size, Cambridge University Press, New York, NY, USA, 1986.

[17] P. Kilham and S. S. Kilham, The Evolutionary Ecology of Phytoplankton, Cambridge University Press, Cambridge, UK, 1980. 

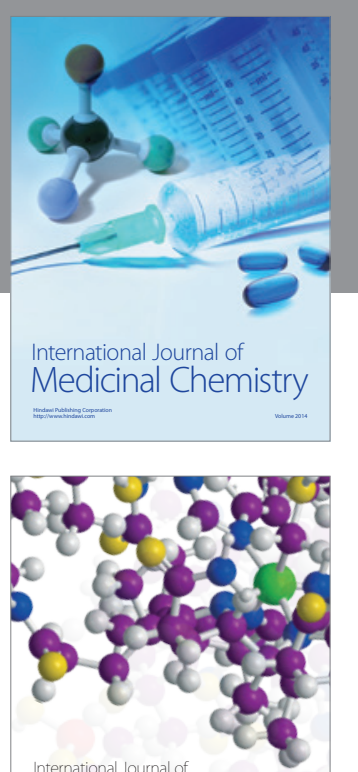

Carbohydrate Chemistry

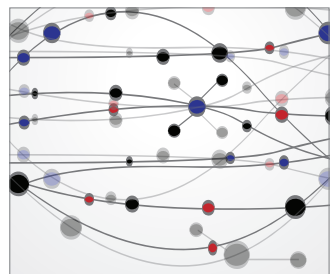

The Scientific World Journal
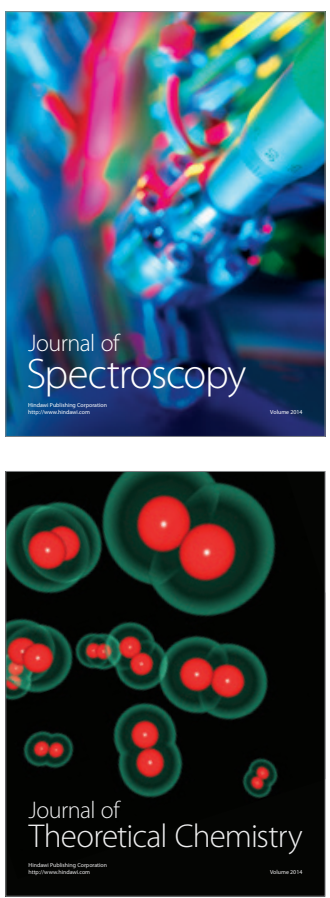
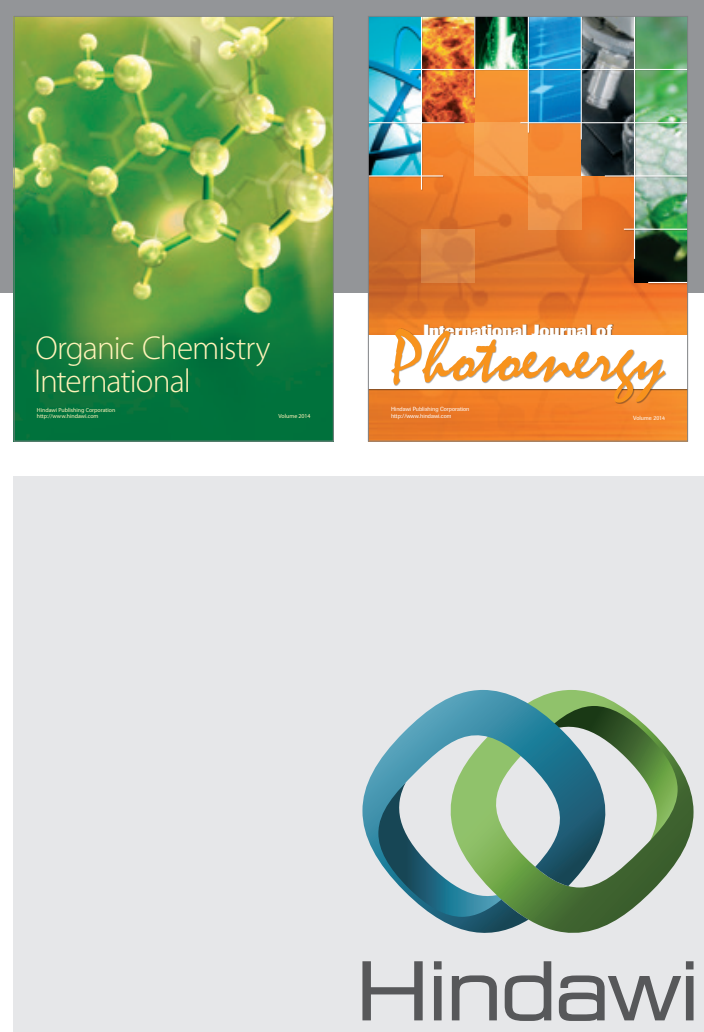

Submit your manuscripts at

http://www.hindawi.com

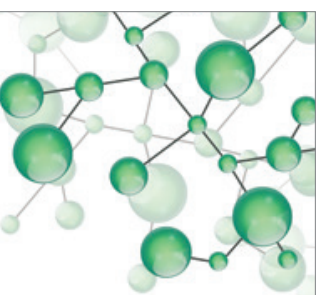

International Journal of

Inorganic Chemistry

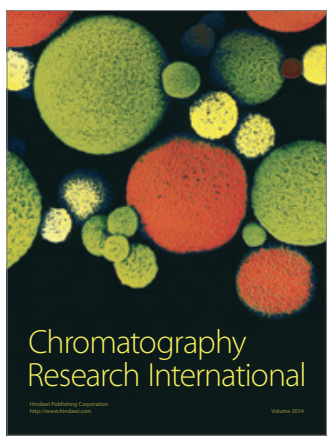

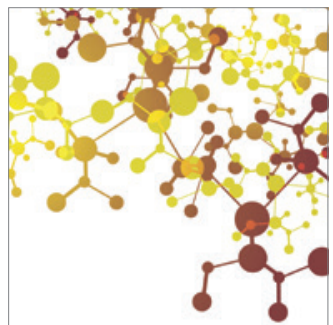

Applied Chemistry
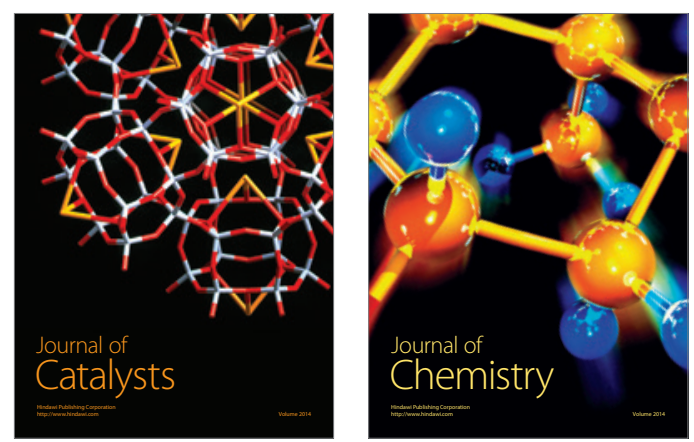
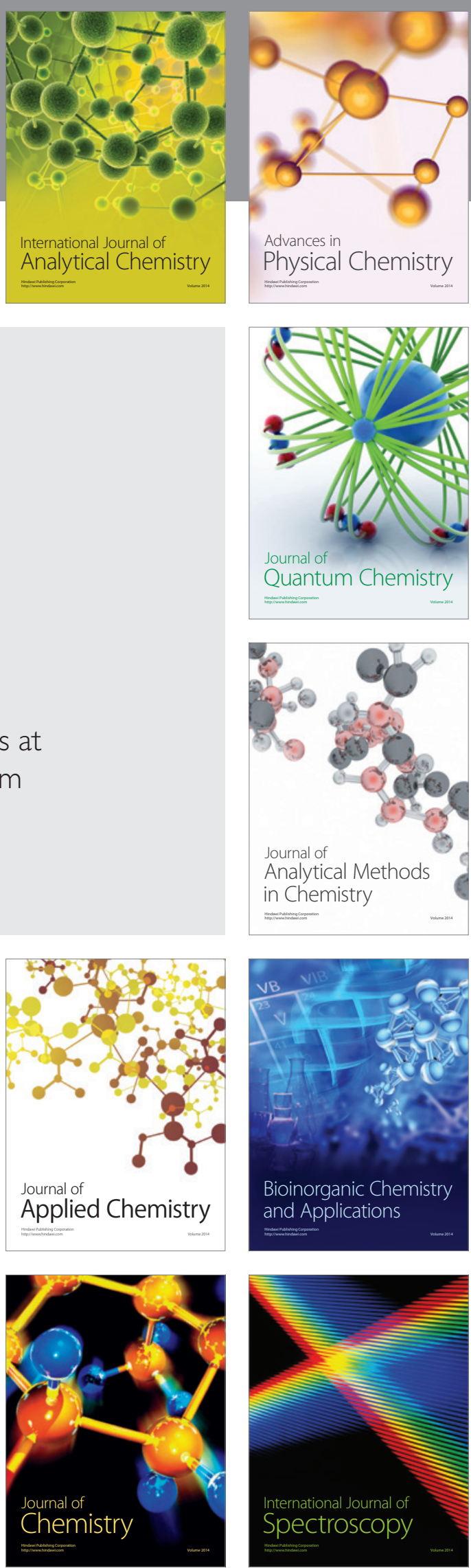